\title{
Lipases efficiently stearate and cutinases acetylate the surface of arabinoxylan films
}

\author{
A.M. Stepan a,b,*, G.E. Anasontzis ${ }^{\mathrm{a}, \mathrm{c}}$, T. Matama $^{\mathrm{d}}$, A. Cavaco-Paulo ${ }^{\mathrm{d}}$, \\ L. Olsson ${ }^{\mathrm{a}, \mathrm{c}}, \mathrm{P}$. Gatenholm ${ }^{\mathrm{a}, \mathrm{b}}$ \\ a WWSC, Wallenberg Wood Science Center, Chalmers University of Technology, Kemivägen 10, SE-412 96 Göteborg, Sweden \\ ${ }^{\mathrm{b}}$ Biopolymer Technology, Department of Chemical and Biological Engineering, Chalmers University of Technology, Kemivägen 10, SE-412 96 Göteborg, \\ Sweden \\ ${ }^{\mathrm{c}}$ Industrial Biotechnology, Department of Chemical and Biological Engineering, Chalmers University of Technology, Kemivägen 10, SE-412 96 Göteborg, \\ Sweden \\ ${ }^{\mathrm{d}}$ IBB - Institute for Biotechnology and Bioengineering, Centre of Biological Engineering, University of Minho, Campus de Gualtar, 4710-057 Braga, Portugal
}

\section{A R T I C L E I N F O}

\section{Article history:}

Received 17 December 2012

Received in revised form 30 May 2013

Accepted 6 June 2013

Available online 14 June 2013

\section{Keywords:}

Hemicellulose

Arabinoxylan

Film

Surface esterification

Enzymatic modification

\begin{abstract}
A B S T R A C T
This is the first report on successful enzyme catalyzed surface esterification of hemicellulose films. Enzyme catalyzed surface acetylation with vinyl acetate and stearation with vinyl stearate were studied on rye arabinoxylan (AX) films. Different surface analytical techniques (FT-IR, TOF-SIMS, ESCA, CA) show that lipases from Mucor javanicus, Rhizopus oryzae and Candida rugosa successfully surface stearate AX films and that a cutinase from Fusarium solani pisi surface acetylates these films. The specificities of cutinase and lipases were also compared, and higher activity was observed for lipases utilizing long alkyl chain substrates while higher activity was observed for cutinase utilizing shorter alkyl chain substrates. The contact angle analysis showed films with increased initial hydrophobicity on the surfaces.
\end{abstract}

(c) 2013 Elsevier B.V. All rights reserved.

\section{Introduction}

Current plastic production is chiefly based on non-renewable resources, and the carbon dioxide emissions resulting from this process and the accumulating non-biodegradable waste are a serious challenge to the ecosystem's balance. Hemicelluloses, being the second most abundant biopolymer on Earth, can make a major contribution toward a sustainable bio-based economy. Besides their potential conversion to biofuels and biochemicals, hemicelluloses and some of their derivatives can be utilized as novel value added bio-based and biodegradable materials such as gels, packaging material, matrices for composites and binders (Ebringerova and Heinze, 2000).

Hemicelluloses are a group of polysaccharides consisting of diverse monosaccharides building up a complex composition and structure (Timell, 1967). In contrast to cellulose, hemicelluloses are nonlinear heteropolymers with a lower molecular weight, which

\footnotetext{
* Corresponding author at: Biopolymer Technology, Department of Chemical and Biological Engineering, Chalmers University of Technology, SE-412 96 Gothenburg, Sweden. Tel.: +46 317723409; fax: +46 317723418 .

E-mail addresses: agnes.stepan@chalmers.se, stepan@chalmers.se(A.M.Stepan).
}

gives them significantly different chemical and material properties compared to cellulose (Tombs and Harding, 1997). For example, arabinoxylan forms films with good oxygen barrier properties, which show a potential as novel packaging materials (Escalante et al., 2011; Grondahl et al., 2004; Hoije et al., 2008). Furthermore, the acetylated arabinoxylans have improved water resistance and attractive mechanical and thermal properties compared to other commercially available bio-based packaging materials (Stepan et al., 2012).

Due to the more complex structure of hemicellulose as compared with cellulose, targeted hemicellulose derivatization is still a challenge. Most of the hemicellulose conversions to date proceed through chemical reactions involving in most cases significant excess of several organic solvents for significant esterification levels (Sun et al., 1999, 2004; Timell, 1967). Surface-only modification, such as the surface octanoylation of starch films, has been developed to decrease the amount of chemicals needed for the conversion (Bengtsson et al., 2003). Enzymes offer an alternative option to carry out surface esterification. Such biocatalytic systems can decrease the amount of chemicals required, many of which can be toxic and dangerous, while enzymes, when immobilized, can be reused, reducing the cost of the enzyme production. Chemical surface modifications may also have high energy demands, with 
reactions taking place at high temperatures, such as $80^{\circ} \mathrm{C}$ in the case of the octanoylation, while most enzymes used in this study have their temperature optimum at around $40^{\circ} \mathrm{C}$. Further advantage of enzymatic surface modifications is that it is less intrusive for the treated material, as they depend on its porosity to access the potential active groups. Enzymes can also provide high level of reaction specificity, which is important for the development of custom-made biomaterials and not always controllable in chemical synthesis systems. Nevertheless, the push toward environmental processes is a main trigger to turn toward enzyme applications for hemicellulose derivatization. The literature on enzyme catalyzed chemical modifications of carbohydrates is continuously expanding. Numerous studies have been done on the activity, stability and specificity of lipases and cutinases in hydrolyzing different ester bonds or transesterification reactions, which have inspired researchers to use lipases and cutinases in a reverse reaction for esterification of hemicelluloses (Kontkanen et al., 2004; Pio and Macedo, 2009; Poulsen et al., 2005; Sakai et al., 2008; Utsugi et al., 2009). It has been shown that some lipases, esterases and cutinases that show activity on esterified substances can also have monosaccharide, oligosaccharide and polysaccharide derivatives as a substrate (Guebitz and Cavaco-Paulo, 2009; Woudenberg-van et al., 1996). The work of (Sakai et al., 2008) and (Utsugi et al., 2009) provides a good overview of some lipases. Their research helped in choosing the lipases from Mucor javanicus, Rhizopus oryzae and Candida rugosa and a cutinase from Fusarium solani pisi in our study. Even though they were selected for their attractive properties, the enzymes used in the presented study have to the best of our knowledge not been applied for esterification of arabinoxylans. Klibanov and co-workers were pioneers in the field of using enzymes in non-aqueous media (Ikeda and Klibanov, 1993; Klibanov, 1989; Patel et al., 1996; Zaks and Klibanov, 1988). While the presence of water is essential in the hydrolysis reaction, esterification on various substrates has been demonstrated using esterases and lipases in non-aqueous media (Chen et al., 2006; Gremos et al., 2011; Kirk et al., 1995; Ljunger et al., 1994; Micaelo et al., 2005; Patel et al., 1996). Enzymatic acylation of polysaccharide derivatives in organic media was also an important landmark in the field, showing activity on not only monomer or oligomer units (Sereti et al., 1998, 2001). Native or unmodified polysaccharides remained beyond the scope of enzymatic functionalization until the last few years (Gremos et al., 2011; Matama et al., 2009). A significant breakthrough was achieved when Gremos et al., 2011 succeeded in performing acylation on cellulose with lipases and a cutinase with different esterifying reagents.

This study is the first report on enzymatic surface esterification of hemicellulose films. The work had two major goals. One of the aims of the project was to substitute the surface of rye arabinoxylan (AX) films in a heterogeneous reaction with a short acyl chain (acetyl) and a long acyl chain (stearate) and to characterize both with respect to chemical modification and possible changes in material properties. The second goal was to investigate and compare the activity of different lipases and a cutinase toward different alkyl chain lengths in esterification reactions of hemicelluloses. By modifying only the surface in a heterogeneous reaction, the reported good oxygen barrier properties of the bulk can be maintained while increasing the hydrophobicity of the film surface (Groendahl et al., 2006; Grondahl et al., 2003; Hoije et al., 2008; Stepan et al., 2012). Electron spectroscopy for chemical analysis (ESCA)/X-ray photoelectron spectroscopy (XPS) provided information about surface acetylation and stearation levels, after which samples (taken after three days of enzymatic surface treatment) were chosen for further analysis. To observe changes on a thinner outer layer of the surfaces, time of flight secondary ion spectroscopy (TOF-SIMS) and Fourier transform infrared (FT-IR) spectroscopy were used. Static contact angle measurements provided information on the hydrophilic and hydrophobic properties of the film surfaces.

\section{Materials and methods}

\subsection{Materials}

Rye arabinoxylan (AX) was purchased from Megazyme (Ireland) ( $\sim 95 \%$ purity, LOT 20601a). Deionized water was used to dissolve and cast the AX films to be treated. The reagents for the enzymatic surface modifications were purchased from Sigma-Aldrich: vinyl-stearate (95\% purity, 43362-08) and vinyl-acetate (V1503). The lipases were also purchased from Sigma-Aldrich: lipase from C. rugosa (L1754), lipase from R. oryzae (86012), lipase from Mucor miehei (L9031) and lipase from M. javanicus (L8906). Cutinase from F. solani pisi was produced as described earlier (Araújo et al., 2007). Hexane (208752) and methanol (32213N) were used to wash the samples after the enzyme treatments.

\subsection{Methods}

\subsubsection{Film preparation}

$1 \mathrm{~g}$ of rye AX was dissolved in deionized water $(40 \mathrm{ml})$. The solution was used to cast films on polystyrene petri dishes. The films were dried in room temperature in a fume hood. The films were freed from the petri dish and cut into $1 \mathrm{~cm}$-wide strips, of which a $2 \mathrm{~cm}$-long portion was hung in the treatment solution. Thus each strip yielded a treated film surface of $4 \mathrm{~cm}^{2}$ for each test specimen.

\subsubsection{Enzymatic surface acylation}

The enzyme loading was as follows: $10 \mathrm{mg}$ of dry enzyme (cutinase or lipase) was suspended in $10 \mathrm{ml}$ of reagent (vinyl acetate or vinyl stearate). A strip of ( $\sim 80 \mu \mathrm{m}$ thick) film with a total surface of $4 \mathrm{~cm}^{2}$ (see above) was kept suspended in the reaction mixture under continuous stirring at $300 \mathrm{rpm}$ and $40^{\circ} \mathrm{C}$ in closed systems to avoid evaporation of the reagents. Separate batches were run for $3 \mathrm{~h}, 6 \mathrm{~h}, 12 \mathrm{~h}, 24 \mathrm{~h}, 72 \mathrm{~h}, 96 \mathrm{~h}$ and $168 \mathrm{~h}$. The samples were then washed several times with methanol and hexane, left to dry in fume hood for $5 \mathrm{~min}$ and stored, covered until surface analysis. Blank samples were treated in pure vinyl acetate (VAc blank) or vinyl stearate (VSt blank) without enzymes for 3 and 7 days. Untreated AX film is also discussed as untreated blank (AX blank), to serve as double blank for both treated blank studies.

\subsubsection{Time-of-flight secondary ion mass spectrometry (TOF-SIMS)}

ToF.SIMS5 from ION-TOF was used and controlled by the Surfacelab 6.1 software for analysis of the outermost one to two monolayers of the modified films. The primary ions used were $\mathrm{Bi}^{3+}$ ions, and the distance to the sample surface was $2 \mathrm{~mm}$ with a primary beam angle of $45^{\circ}$ with respect to the surface. The area analyzed was selected manually $(\sim 100 \mu \mathrm{m} \times 100 \mu \mathrm{m})$, and the spectra were recorded for $50 \mathrm{~s}$ in high current bunched mode. A flood gun was used for charge compensation. Measurements were made in both positive and negative modes.

\subsubsection{Fourier transform infrared spectroscopy (FT-IR)}

The surface of the AX films was scraped off with a scalpel, and this powder was mixed with $\mathrm{KBr}$ to press tablets. The samples were analyzed with a System 2000 FT-IR from Perkin Elmer controlled by Spectrum software version 5.3 in transmission mode using a triglycine sulphate (TGS) detector. Twenty scans were taken with a resolution of $4 \mathrm{~cm}^{-1}$. Samples were also run in reflection absorption spectra (RAS) mode on a Spectratech FT-80 with a liquid nitrogen-cooled mercury cadmium telluride (MCT) detector. In this mode, 200 scans were taken with a resolution of $4 \mathrm{~cm}^{-1}$. The 


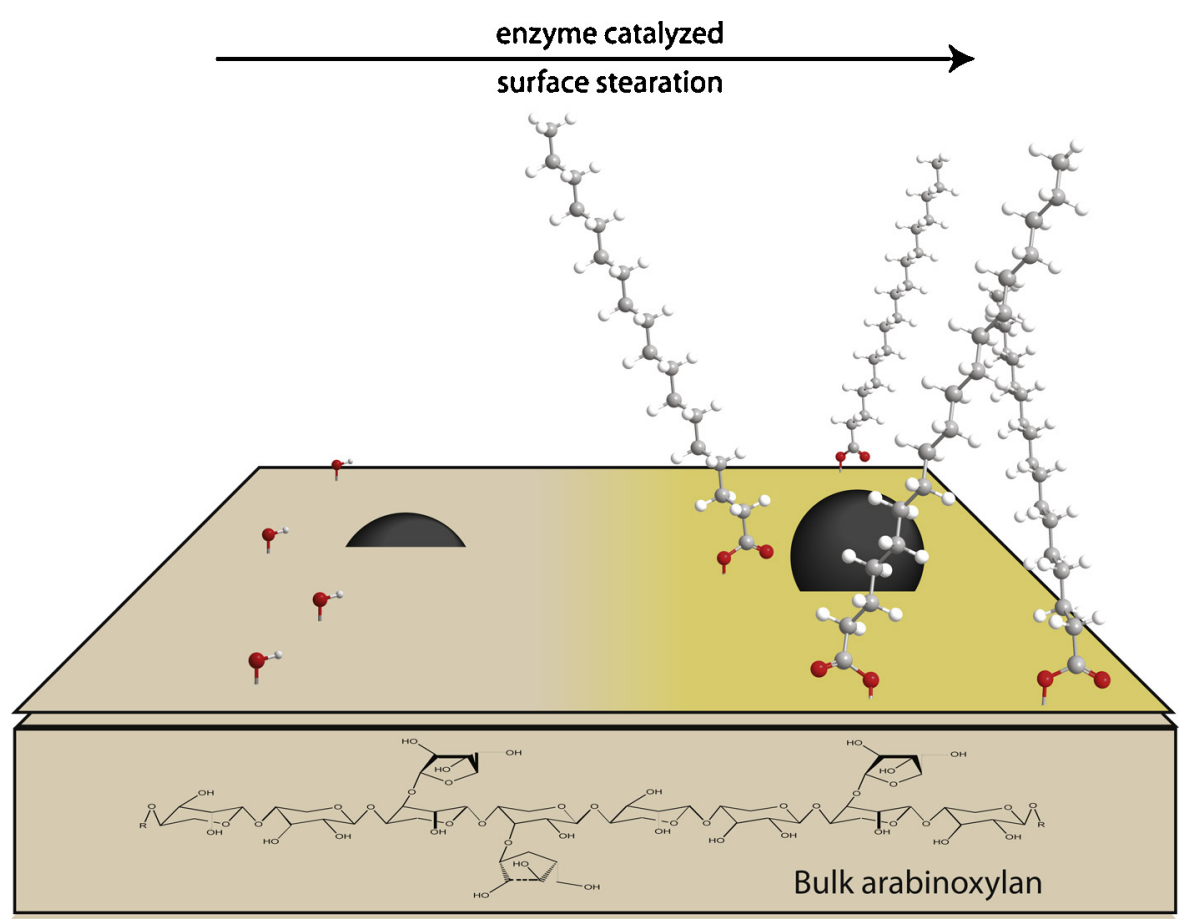

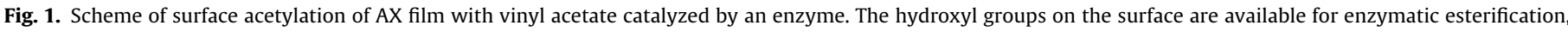
while the hydroxyl groups in the bulk of the material are not available.

incident angle was near grazing angle $\left(85^{\circ}\right)$ with non polarized IR. The background was run on a quartz crystal microbalance (QCM) (from Qsense, QSX301) gold crystal. For the RAS mode, the samples were prepared as follows: AX films were drop-casted from $2.5 \%$ deionized water solution onto QCM gold crystals and, after drying, they were enzymatically surface acylated. After washing these samples in the same way as the films described above, the films were allowed to dry and were analyzed in RAS mode.

\subsubsection{Electron spectroscopy for chemical analysis (ESCA)/X-ray photoelectron spectroscopy (XPS)}

Surface composition analysis was done on a Quantum 2000 scanning ESCA microprobe from Physical Electronics controlled by Multipack software. An Al K $\alpha$ (1486.6 eV) X-ray source was used, and the beam size was $100 \mu \mathrm{m}$. The area analyzed was selected manually $(\sim 500 \mu \mathrm{m} \times 500 \mu \mathrm{m})$, and the take-off angle was $45^{\circ}$ with respect to the sample surface. The information depth is approximately 4-5 $\mathrm{nm}$.

\subsubsection{Contact angle}

Advancing contact angle against water was measured in a static contact angle measuring mode on an Attention device, model $\Theta$. Pictures were taken with a camera once every second for $30 \mathrm{~s}$ for each measurement. The collected data was analyzed by Attention $\Theta$ software. The highest initial contact angle is reported.

\section{Results and discussion}

\subsection{Enzymatic surface esterification}

In the present study, the enzymatic surface esterification of arabinoxylan was performed in the absence of water in order to favor the esterification direction of the reaction. Since the activities of the enzymes for the esterification reactions were unknown, the enzyme load was uniformly set to $10 \mathrm{mg}$ of freezedried enzyme in each experiment. AX was insoluble in vinyl acetate or in vinyl stearate; this resulted in heterogeneous reaction systems, which are ideal for surface modifications. The enzymatic surface acylation of an AX film is schematically illustrated in Fig. 1.

In a heterogeneous reaction, it is reasonable to believe that the enzyme will access more easily hydroxyl groups that are available on the surface of the film. Various analytical methods (ESCA, FT-IR, TOF-SIMS, CA) with different sensitivities and information depths on the analyzed sample surfaces were applied to confirm the successful acetylation or stearation. In the first screening analysis of the modified films, an optimal treatment time for further analysis was selected based on ESCA results. It was found that three days of reaction provided a set of samples that allowed the comparison of the different reactions. TOF-SIMS, FT-IR and contact angle measurements were consequently performed on the samples that were enzymatically surface treated for 3 days. Results from the ESCA are shown for the 3 and 7 day samples to present consistency of the experiments.

\subsection{TOF-SIMS}

The TOF-SIMS is a highly surface sensitive analytical method. The information depth is $1 \mathrm{~nm}$, which allows a more accurate composition analysis of the outermost molecular layer. The method is not quantitative, but provides supporting data on surface composition. Spectra of the VSt blank for 3 days are presented with the sample which was treated for 3 days in vinyl stearated in the presence of M. javanicus (Fig. 2). The spectral evaluation was straightforward for the stearated samples, since a characteristic signal group of the stearate molecule and its fragments were identified on the positive scan at 329 mass units (Fig. 2a) and on the negative scan at 283 mass units (Fig. 2b and c). As TOF-SIMS was not useful for identifying acetylation, other methods were applied for those samples.

\subsection{FT-IR}

Three different FT-IR techniques were used for characterization of the surface modified samples: two types of transmission modes 
a)

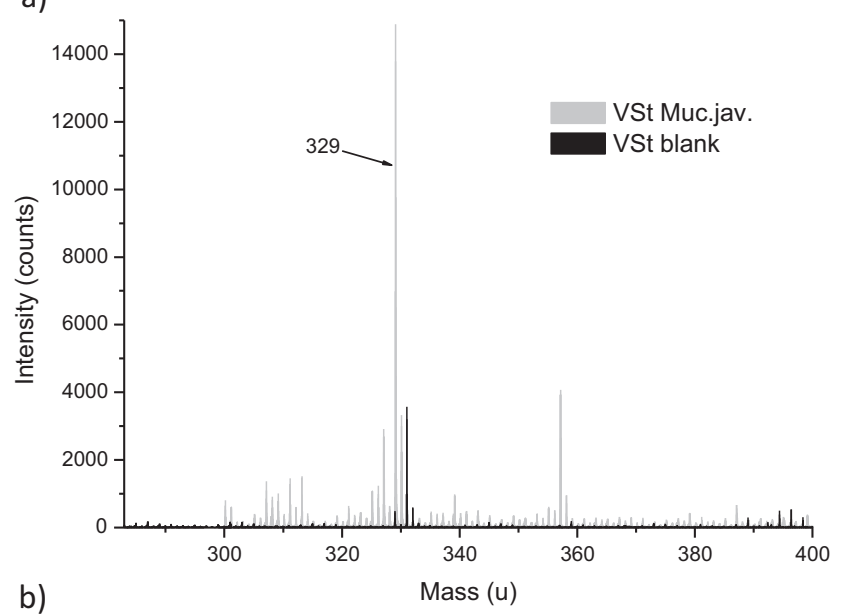

b)

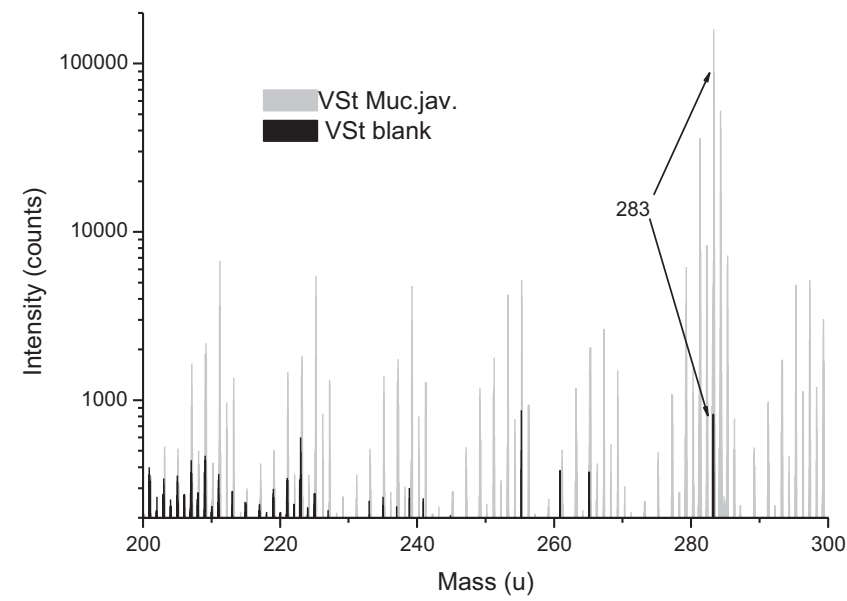

Fig. 2. (a) Positive TOF-SIMS scan of VSt blank film surface and enzymatically surface stearated films (with Mucor javanicus) (300-400 mass units); (b) VSt blank film surface and enzymatically surface stearated films (with M. javanicus) (200-300 mass units) shown on logarithmic $y$ axis for better visualization of the VSt blank.

(one by preparing $\mathrm{KBr}$ tablets and one by using a free-standing thin film with a beam condenser) and a RAS mode. The $\mathrm{KBr}$ tablet contained the scratched off surface of the films, as well as some deeper unacylated molecular layers, resulting in a weak signal at the FT-IR (Fig. 3). Fig. 3a presents the untreated blank sample with the blank sample that was treated in vinyl acetate and the blank treated in vinyl stearate for 3 days. A signal at $1752 \mathrm{~cm}^{-1}$ indicates some carbonyl groups, but no relevant signal can be identified at $2918 \mathrm{~cm}^{-1}$, which suggest no significant amount of acetyl groups on the surface. The VSt blank does not show characteristic signals of acylation compared to the AX blank. The spectra of the enzyme treated samples clearly show signals originating from the carbonyl group of the ester bonds appearing for all treated samples at $1752 \mathrm{~cm}^{-1}$ and from the alkyl chains at $2918 \mathrm{~cm}^{-1}$ also compared to their respective blanks (Fig. 3b and c). Using thin free films, very similar data were obtained for the method using $\mathrm{KBr}$ tablets (not shown). The reflection absorption spectra of the samples showed very clear and distinct peaks for the carbonyl $\left(1752 \mathrm{~cm}^{-1}\right)$ and the alkyl groups $\left(2918 \mathrm{~cm}^{-1}\right)$ (Fig. 3b). These were significantly more pronounced than for the technique with $\mathrm{KBr}$ tablets. The results of both FT-IR techniques demonstrate clear proof of surface acylation of the films.

\subsection{ESCA/XPS}

The ESCA spectra provide elemental surface composition of samples, and high resolution spectra also give information on the chemical bonds between the elements. The peaks from right to left correspond to carbon bound only to carbon and hydrogen (C1), carbon with one bond to oxygen (C2), carbon with two bonds to oxygen(s) (C3) and carbon with three bonds to oxygens (C4) (Groendahl et al., 2006).

There are many signs of the presence of residual proteins in the pure starting material. The atomic concentration table and the survey spectrum showed nitrogen content of $4.1 \%$ in the sample. Annual plants, such as rye, have a significant amount of proteins in the cell walls, which means amino acids can probably be detected in hemicellulose extracts that are not $100 \%$ pure (Albersheim et al., 2011). That the oxygen to carbon ratio is 0.57 instead of 0.8 also indicates the presence of non-polysaccharide components in the sample. The spectrum of pure AX should not contain C1 and C4 peaks, but the traces of proteins and non polysaccharide residues together could give an explanation for the presence of both the $\mathrm{C1}$ peak and the $C 4$ peak. Without the presence of protein in the AX, the acylation of the samples could be determined by the appearance of $\mathrm{C} 1$ (the $\mathrm{C}-\mathrm{C}$ bonds of the alkyl chains) and $\mathrm{C} 4$ (showing the presence of carboxylate groups) in the esterified samples. Full esterification would be indicated by the ratio of the areas of $\mathrm{C} 4 / \mathrm{C} 3$ peaks being two for pure samples. Hence the existence of the $\mathrm{C} 1$ and $\mathrm{C} 4$ peaks in the blank AX sample, the relative increase of the $\mathrm{C} 1$ peak compared to the other peaks will indicate the new, non-protein originated, $\mathrm{C}-\mathrm{C}$ bonds from the alkyl chains; and the increased ratio of the $\mathrm{C} 4 / \mathrm{C} 3$ peaks will indicate new carbonyl groups from the ester bond on the surface.

Due to the presence of non-polysaccharide components, no quantitative conclusion could be made from C4/C3. Furthermore, the ESCA information depth is $4-5 \mathrm{~nm}$, which is deeper than the thickness of the outermost molecular layer, and the information collected therefore also corresponds to molecular layers under the surface that were not accessible to the enzymes. Thus, in this case, the method is not surface sensitive enough to determine reaction yields or degrees of substitution, but changes on the surface can be followed.

The ESCA high resolution carbon peak of the AX blank can be seen in Fig. 4a as serving as a double blank for both of the treated blank samples. The treated blank samples were treated in pure vinyl acetate or vinyl stearate without enzymes for three days, Fig. 4b and $d$, respectively. These blanks were evaluated for adsorption of the reagent. The VAc blanks represented some acetate deposition on the surface based on the increase of the $\mathrm{C} 4 / \mathrm{C} 3$ ratio (Table $1 \mathrm{a}$ ). The VSt blanks showed a higher C1 peak as compared to the AX blank samples, but no difference was seen in the $\mathrm{C} 4 / \mathrm{C} 3$ ratio. This means there may be some adsorbed stearate on the surface but that no ester bonds were formed (Table $1 \mathrm{~b}$ ). This can be caused by the fact that the vinyl stearate is solid in room temperature and, even though washing of the samples at the end of the treatment was done with warm methanol and hexane, there is a chance of some stearate solidifying on the surface of the films. The enzyme treated samples were therefore compared to their respective treated blanks.

The ESCA spectrum of an AX film after 3 days of acetylation with cutinase from $F$. solani pisi (Fig. 4d) and after 3 days of stearation with lipase from M. javanicus (Fig. 4e) showed successful acylation with both an increased $\mathrm{C} 1$ signal and an increased ratio of $\mathrm{C} 4 / \mathrm{C} 3$ compared to their respective treated blanks. The other two lipases (from C. rugosa and $R$. oryzae) showed activity similar to that of the lipase presented in Fig. 4e. The measurements from the samples treated for 7 days were in good agreement with the ones treated for 3 days, detecting similar or slightly increased levels of esterification (Table 1). The lower nitrogen content of the successfully enzyme treated samples confirms that no enzyme is adsorbed to the surface of the film. The amount of nitrogen detected by ESCA is lower than for the AX blank, since the composition of the detected top $4-5 \mathrm{~nm}$ on the surface should have increased oxygen and 
a)
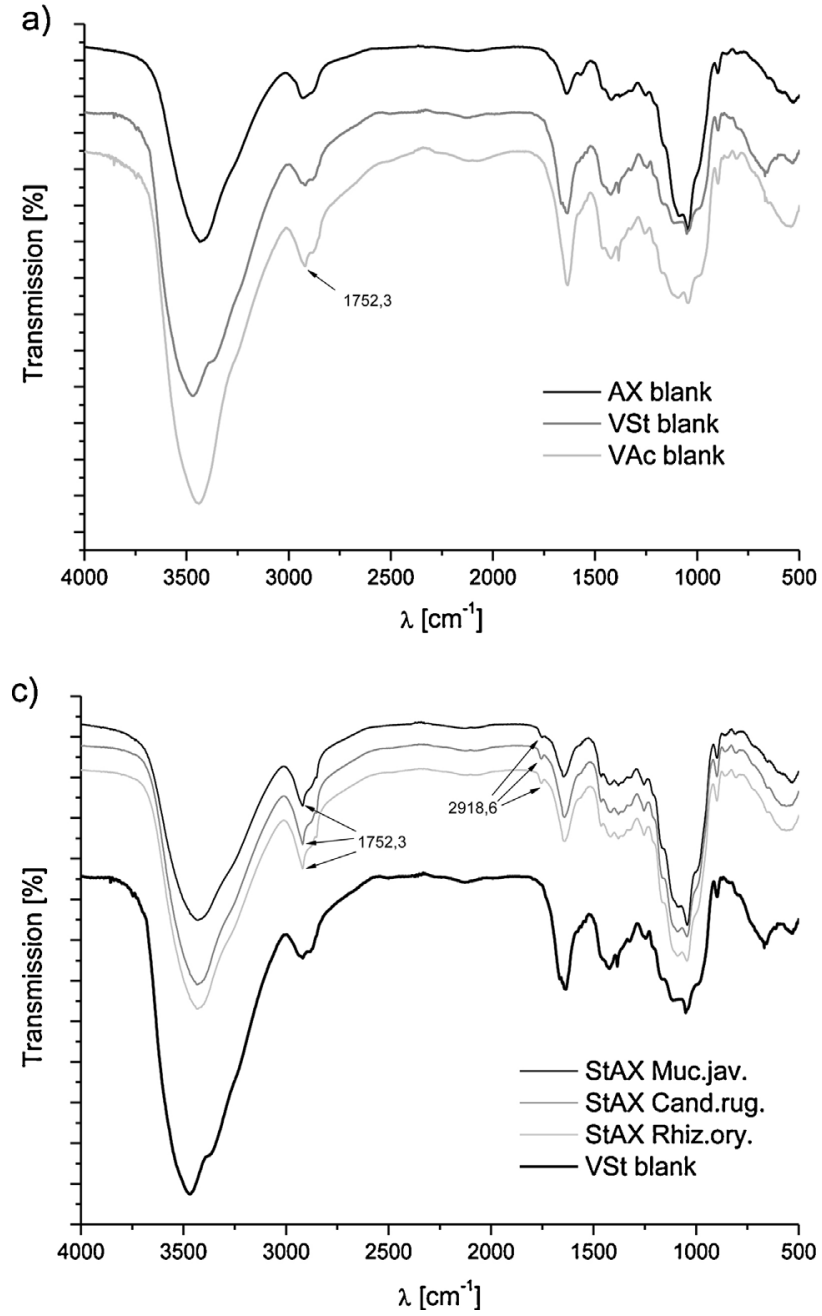
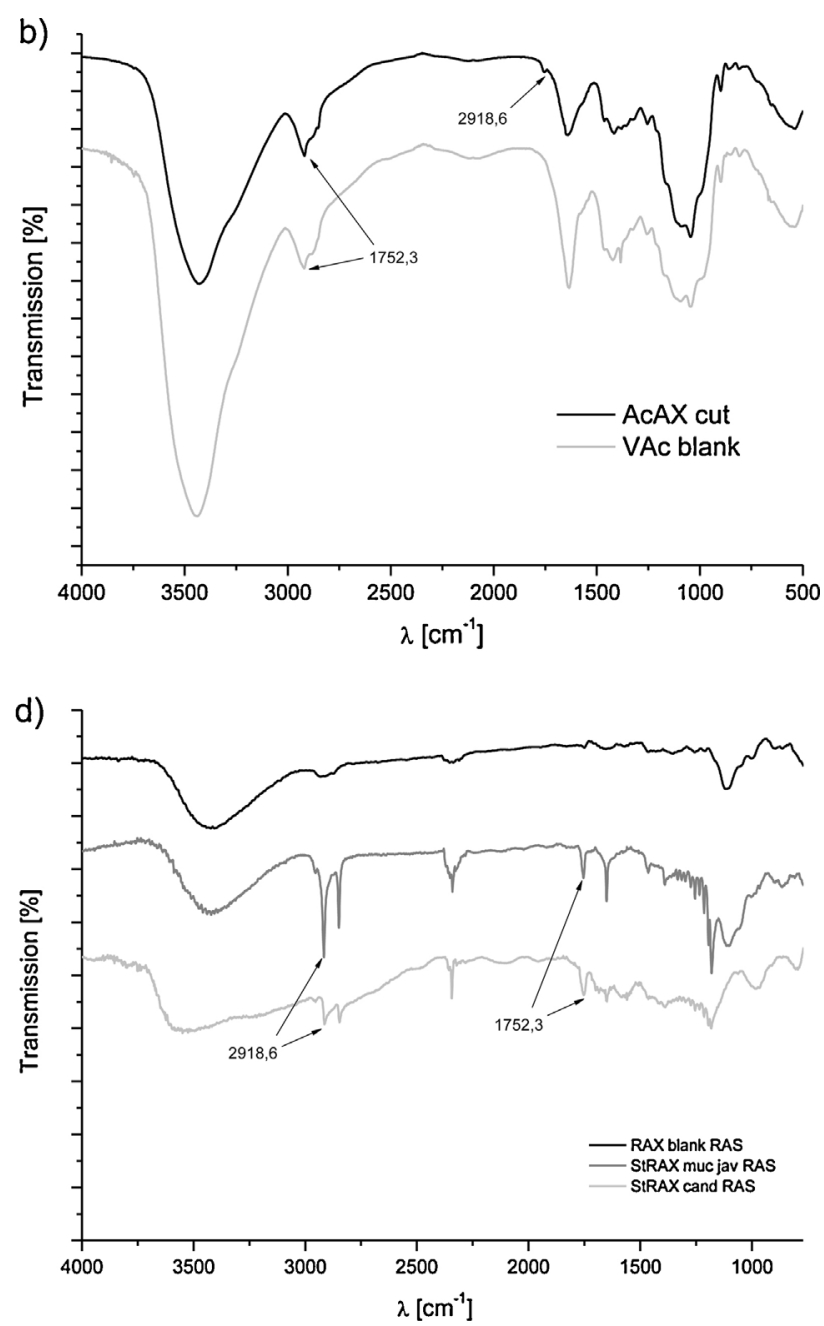

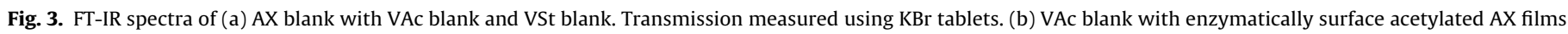

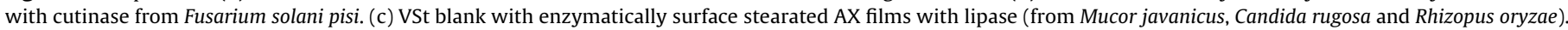
(d) FT-IR spectra of AX blank with enzymatically stearated AX films (lipase from Mucor javanicus and Candida rugosa) run in RAS mode.

carbon after acylation, covering some nitrogen, thus decreasing the detected proportion of it. This was confirmed in all successfully surface acylated samples compared to any blank sample. The detected ester bonds and $\mathrm{C}-\mathrm{C}$ bonds are therefore from the acetates and stearates esterified on the surface of the film.
Often, esterases are distinguished from lipases by their higher activity for hydrolyzing short alkyl chain esters than for hydrolyzing long alkyl chain esters (in aqueous media). In this study it was expected to see similar activities in the reverse reaction in non aqueous media. We ran separate experiments with all the enzymes and with both short (vinyl acetate) and long chain (vinyl stearate)

Table 1

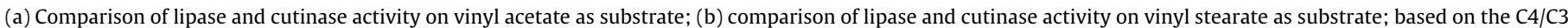
ratio from ESCA (where the C4/C3 for the untreated blank is 0.13 ).

\begin{tabular}{|c|c|c|c|}
\hline Enzyme & Enzyme origin & Acetylation 3 days & Acetylation 7 days \\
\hline Lipase from Mucor javanicus & (Sigma) L8906 & 0.13 & 0.23 \\
\hline Lipase from Candida rugosa & (Sigma) L1754 & 0.12 & 0.23 \\
\hline Lipase from Rhizopus oryzae & (Sigma) 86012 & 0.22 & 0.22 \\
\hline Cutinase from Fusarium solani pisi & Research enzyme & 0.80 & 0.80 \\
\hline Respective treated VAc blank & None & 0.21 & 0.26 \\
\hline \multicolumn{4}{|l|}{ (b) } \\
\hline Enzyme & Enzyme origin & Stearation 3 days & Stearation 7 days \\
\hline Lipase from Mucor javanicus & (Sigma) L1754 & 0.22 & 0.34 \\
\hline Lipase from Candida rugosa & (Sigma) 86012 & 0.18 & 0.34 \\
\hline Lipase from Rhizopus oryzae & (Sigma) L9031 & 0.32 & 0.30 \\
\hline Cutinase from Fusarium solani pisi & Research enzyme & 0.21 & n.a. \\
\hline Respective treated VSt blank & None & 0.12 & 0.14 \\
\hline
\end{tabular}




\section{a)}

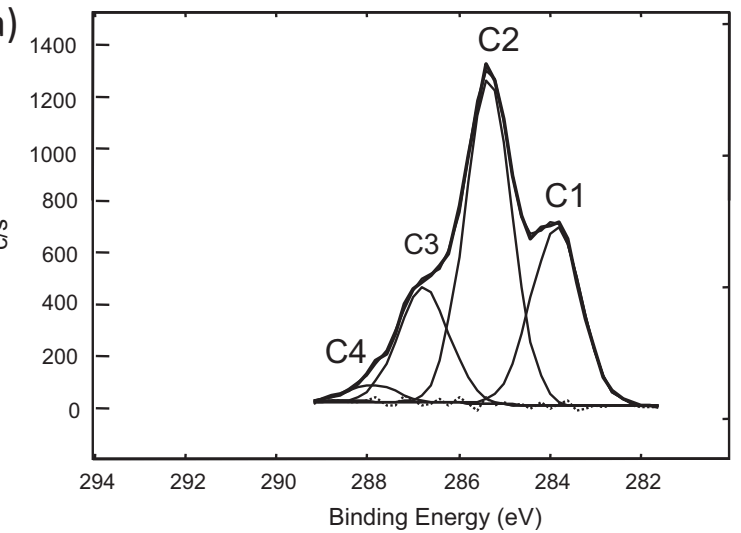

c)

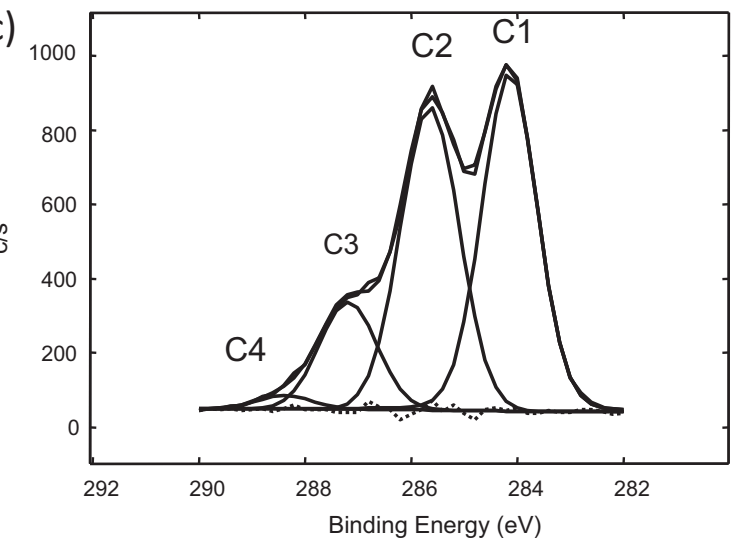

b)

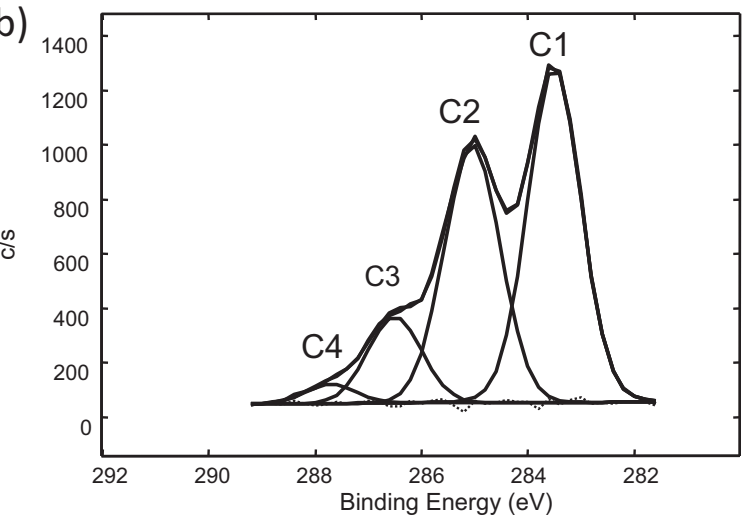

d)

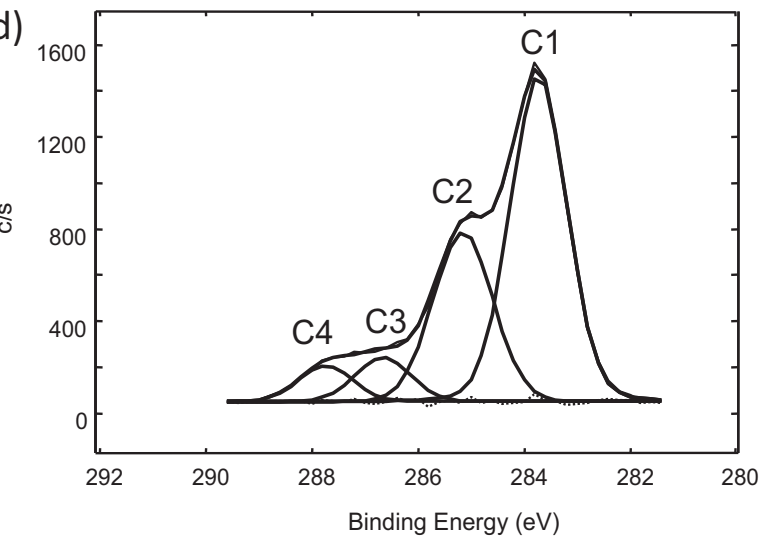

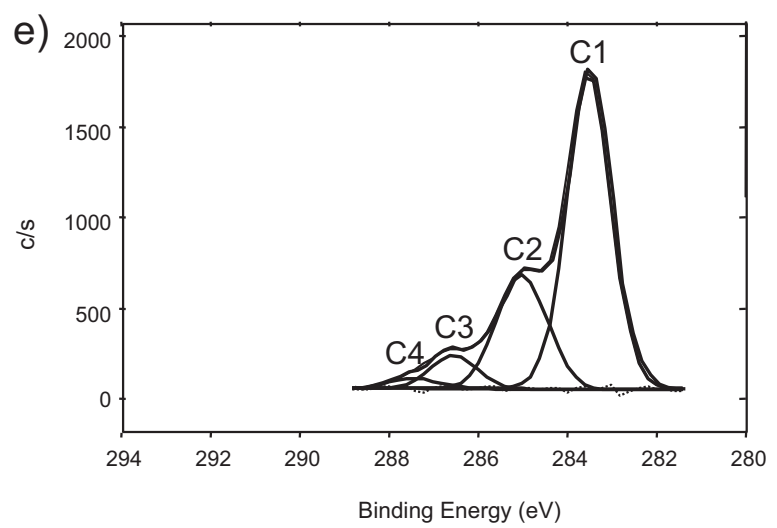

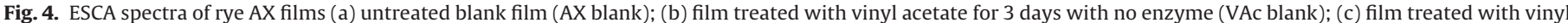

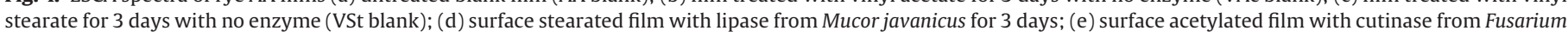
solani pisi for 3 days.

substrates, and our findings on activity differences of lipases and cutinases toward different substrates are summarized in Table 1.

A general conclusion is that lipases work efficiently for stearation, whereas cutinase is more efficient for acetylation but also showed activity when vinyl stearate was used as reagent for stearation. None of the lipases from M. javanicus and R. oryzae showed activity on AX films when vinyl acetate was used for acetylation. A significant conclusion is that the cutinase resulted in mentionably higher substitution levels of acetylation than the lipases for stearation (based on the increase in $\mathrm{C} 4 / \mathrm{C} 3$ ratios). The reason for this might be that, in most reports, cutinase has no hydrophobic lid, and the enzyme does not require a water-lipid interface for its activity in general, while the lipases used in the experiments all had the hydrophobic lid structure (Martinez et al., 1992; Maruyama et al., 2003). It is important to note for the commercially available lipase from C. rugosa that it is a mix of isoenzymes with different specificities (Akoh et al., 2004). It has also been reported that the reproducibility of reactions is not satisfactory with C. rugosa lipase due to the variable proportion of isoforms, amount of water in the crude lyophilized lipase and the amount of lipolytic protein present in the powdered enzyme (Dominguez and Sinisterra, 1999). Interestingly, this enzyme had lower activity and a notable deviation between repeated batches compared to the other lipases. This limits the application of the crude lipase in the reproducible production of specific compounds with high purity. 

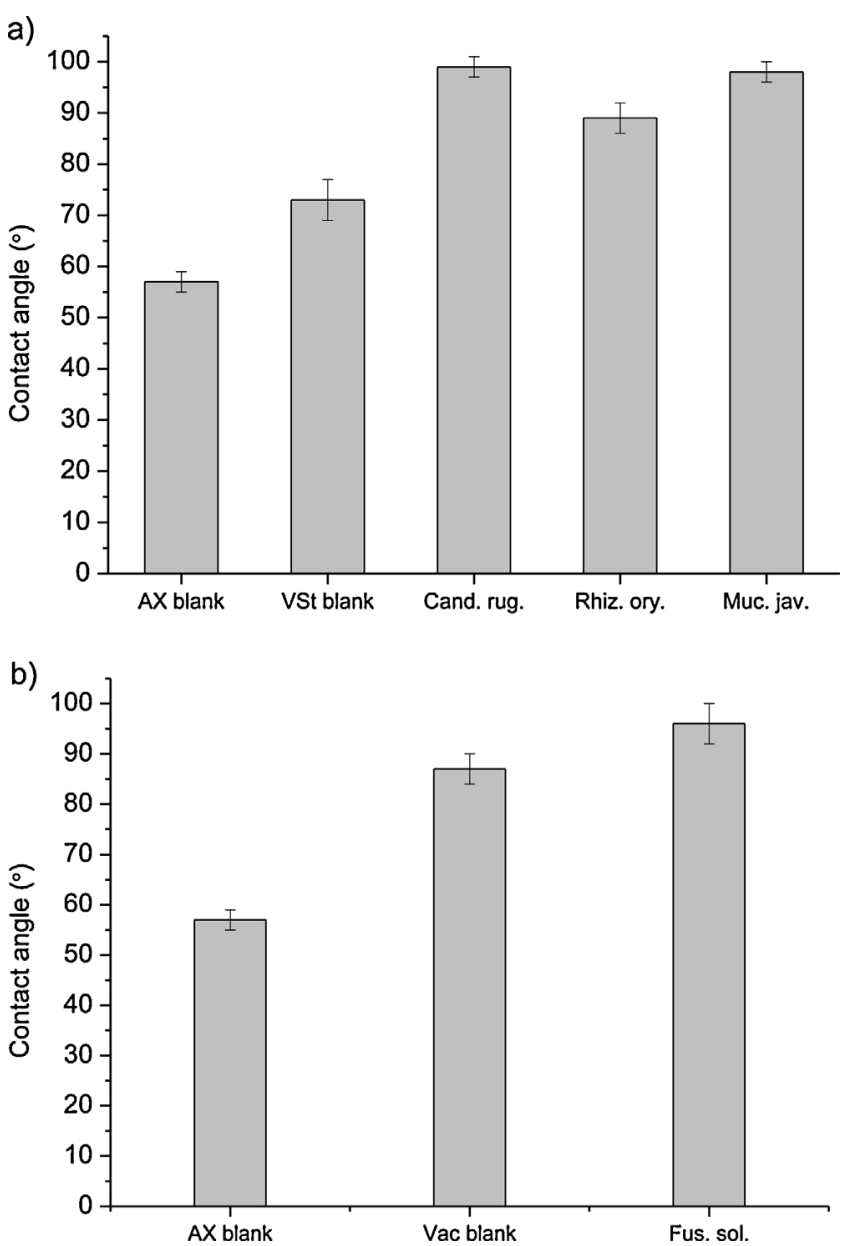

Fig. 5. Contact angle against water of (a) enzymatically surface stearated and (b) enzymatically surface acetylated AX films.

\subsection{Contact angle}

Xylan films are hydrophilic, while their acetylated derivatives are less hydrophilic (Escalante et al., 2011; Grondahl et al., 2003; Stepan et al., 2012). The untreated blank AX films have an advancing contact angle of $57^{\circ}$, which increased to $87^{\circ}$ for the VAc blank and to $73^{\circ}$ for the VSt blank samples. The ESCA, ToF-SIMS and FT-IR did not detect stearate esters on the surface of the VSt blank. However it was also noted that traces of deposition on the surface are possible, which could also have a strong influence on the measured contact angle despite them not being covalently bound and at low quantities. For the VAc blank, the ESCA showed signs of ester bonds, thus the contact angle increase is not a surprise for the treated blank. The contact angle measurement resulted in an increased advancing contact angle for all enzymatically surface modified films compared to their treated respective blanks. The averages and standard deviations of the results are shown in Fig. 5. After about 20-30 s, the contact angle started to decrease significantly for all samples and the films dissolved with time under the droplet. A possible explanation is that a very thin monolayer of acyl substitution was achieved, and a water induced molecular rearrangement of the surface occurred (Andrade et al., 1983). The bulk of the film is still rich in hydroxyl groups, which are obviously more hydrophilic. Due to the molecular mobility of the surface, the more hydrophilic hydroxyl groups can turn toward the surface, come into contact with the aqueous layer and become hydrated, thus slowly allowing water to penetrate the whole film. Since the acylation of the surface of the films does not affect the bulk material, the good oxygen barrier properties, which are characteristic for non-acetylated xylans, should be retained (Escalante et al., 2011; Hoije et al., 2008). The increased hydrophobicity of the surface could provide an opportunity to produce laminates with good adhesion to hydrophobic films (based on the chemical similarities of the surfaces).

\section{Conclusions}

Enzymatic surface esterification of arabinoxylan films was demonstrated for the first time. Different enzymes were evaluated for surface acetylation and stearation of rye arabinoxylan (AX) films. The reactants (vinyl acetate and vinyl stearate) also served as the reaction media, making the reaction a solvent free reaction. Surface analysis showed that lipases from M. javanicus, R. oryzae and $C$. rugosa successfully surface stearated, and a cutinase from $F$. solani pisi surface acetylated the AX films after 3 days of reaction time.

\section{Acknowledgements}

Anne Wendel, Anders Mårtensson and Per Malmberg from Chalmers University of Technology are acknowledged for their help in the ESCA, FT-IR and SIMS measurements. The Knut and Alice Wallenberg Foundation is gratefully acknowledged for donating a base for a Swedish Research Center, the Wallenberg Wood Science Center, thus financing this work.

\section{References}

Akoh, C.C., Lee, G.-C., Shaw, J.-F., 2004. Protein engineering and applications of Candida rugosa lipase isoforms. Lipids 39, 513-526.

Albersheim, P., Darvill, A., Roberts, K., Sederoff, R., Staehelin, A., 2011. Plant Cell Walls. Garland Science. Taylor \& Francis Group, LLC, New York, USA/Abingdon, UK.

Andrade, J.D., Gregonis, D.E., Smith, L.M., 1983. Polymer-water interface dymanics. In: Mittal, K.L.(Ed.), Physico-Chemical Aspects of PolymerSurfaces. Plenum, New York, pp. 911-922.

Araújo, R., Silva, C., O’Neill, A., Micaelo, N., Guebitz, G., Soares, C.u.M., Casal, M. Cavaco-Paulo, A., 2007. Tailoring cutinase activity towards polyethylene terephthalate and polyamide 6,6 fibers. Journal of Biotechnology 128, 849-857.

Bengtsson, M., Koch, K., Gatenholm, P., 2003. Surface octanoylation of high-amylose potato starch films. Carbohydrate Polymers 54, 1-11.

Chen, Z.-G., Zong, M.-H., Li, G.-J., 2006. Lipase-catalyzed acylation of konjac glucomannan in ionic liquids. Journal of Chemical Technology and Biotechnology 81 $1225-1231$.

Dominguez, D.M.P., Sinisterra, G.J.V., 1999. Causes of unreproducibility of C. rugosa lipase-catalyzed reactions in slightly hydrated organic media. Tetrahedron 55 8555-8566.

Ebringerova, A., Heinze, T., 2000. Xylan and xylan derivatives - biopolymers with valuable properties. 1 . Naturally occurring xylans structures, isolation procedures and properties. Macromolecular Rapid Communications 21, 542-556.

Escalante, A., Goncalves, A., Bodin, A., Stepan, A., Sandstroem, C., Toriz, G., Gatenholm, P., 2011. Flexible oxygen barrier films from spruce xylan. Carbohydrate Polymers 87, 2381-2387.

Gremos, S., Zarafeta, D., Kekos, D., Kolisis, F., 2011. Direct enzymatic acylation of cellulose pretreated in BMIMCl ionic liquid. Bioresource Technology 102 1378-1382.

Groendahl, M., Gustafsson, A., Gatenholm, P., 2006. Gas-phase surface fluorination of arabinoxylan films. Macromolecules 39, 2718-2721.

Grondahl, M., Eriksson, L., Gatenholm, P., 2004. Material properties of plasticized hardwood xylans for potential application as oxygen barrier films. Biomacromolecules 5, 1528-1535.

Grondahl, M., Teleman, A., Gatenholm, P., 2003. Effect of acetylation on the material properties of glucuronoxylan from aspen wood. Carbohydrate Polymers 52, 359-366.

Guebitz, G.M., Cavaco-Paulo, A., 2009. Enzymes go big: surface hydrolysis and functionalization of synthetic polymers. American Chemical Society, POLY-175.

Hoije, A., Sternemalm, E., Heikkinen, S., Tenkanen, M., Gatenholm, P., 2008. Material properties of films from enzymatically tailored arabinoxylans. Biomacromolecules 9, 2042-2047.

Ikeda, I., Klibanov, A.M., 1993. Lipase-catalyzed acylation of sugars solubilized in hydrophobic solvents by complexation. Biotechnology and Bioengineering 42 , 788-791.

Kirk, O., Christensen, M.W., Beck, F., Damhus, T., 1995. Lipase-catalyzed regioselective acylation and deacylation of glucose derivatives. Biocatalysis and Biotransformation 12, 91-97. 
Klibanov, A.M., 1989. Enzymatic catalysis in anhydrous organic solvents. Trends in Biochemical Sciences 14, 141-144.

Kontkanen, H., Tenkanen, M., Fagerstrom, R., Reinikainen, T., 2004. Characterisation of steryl esterase activities in commercial lipase preparations. Journal of Biotechnology 108, 51-59.

Ljunger, G., Adlercreutz, P., Mattiasson, B., 1994. Lipase catalyzed acylation of glucose. Biotechnolgy Letters 16, 1167-1172.

Martinez, C., De Geus, P., Lauwereys, M., Matthyssens, G., Cambillau, C., 1992. Fusarium solani cutinase is a lipolytic enzyme with a catalytic serine accessible to solvent. Nature 356, 615-618.

Maruyama, T., Nakajima, M., Kondo, H., Kawasaki, K., Seki, M., Goto, M., 2003. Can lipases hydrolyze a peptide bond? Enzymes and Microbial Technology 32 , 655-657.

Matama, T. Araujo, R., Gubitz, G.M., Casal, M., Cavaco-Paulo, A., 2009. Functionalization of cellulose acetate fibers with engineered cutinases. Biotechnology Progress 26, 636-643.

Micaelo, N.M., Teixeira, V.H., Baptista, A.M., Soares, C.M., 2005. Water dependent properties of cutinase in nonaqueous solvents: a computational study of enantioselectivity. Biophysical Journal 89, 9991008.

Patel, M.T., Nagarajan, R., Kilara, A., 1996. Lipase-catalyzed biochemical reactions in novel media. A review. Chemical Engineering Communications 152/153, 365-404.

Pio, T.F., Macedo, G.A., 2009. Cutinases: properties and industrial applications. Advances in Applied Microbiology 66, 77-95.

Poulsen, K.R., Snabe, T., Petersen, E.I., Fojan, P., Neves-Petersen, M.T., Wimmer, R., Petersen, S.B., 2005. Quantization of pH: evidence for acidic activity of triglyceride lipases. Biochemistry 44, 11574-11580.
Sakai, S., Antoku, K., Yamaguchi, T., Kawakami, K., 2008. Transesterification by lipase entrapped in electrospun poly(vinyl alcohol) fibers and its application to a flowthrough reactor. Journal of Bioscience and Bioengineering 105, 687-689.

Sereti, V., Stamatis, H., Koukios, E., Kolisis, F.N., 1998. Enzymic acylation of cellulose acetate in organic media. Journal of Biotechnology 66, 219-223.

Sereti, V., Stamatis, H., Pappas, C., Polissiou, M., Kolisis, F.N., 2001. Enzymatic acylation of hydroxypropyl cellulose in organic media and determination of ester formation by diffuse reflectance infrared fourier transform (DRIFT) spectroscopy. Biotechnology and Bioengineering 72, 495-500.

Stepan, A.M., Hoeije, A., Schols, H.A., de, W.P., Gatenholm, P., 2012. Arabinose content of arabinoxylans contributes to flexibility of acetylated arabinoxylan films. Journal of Applied Polymer Science (ahead of print).

Sun, R., Fang, J.M., Tomkinson, J., Jones, G.L., 1999. Acetylation of wheat straw hemicelluloses in $\mathrm{N}, \mathrm{N}$-dimethylacetamide/LiCl solvent system. Industrial Crops and Products 10, 209-218.

Sun, R., Sun, X.F., Tomkinson, J., 2004. Hemicelluloses and their derivatives. ACS Symposium Series 864, 2-22.

Timell, T.E., 1967. Recent progress in the chemistry of wood hemicelluloses. Wood Science and Technology 1, 45-70.

Tombs, M., Harding, S., 1997. An Introduction to Polysaccharide Biotechnology. Taylor \& Francis, London, UK/Bristol PA, USA.

Utsugi, A., Kanda, A., Hara, S., 2009. Lipase specificity in the transacylation of triacylglycerin. Journal of Oleo Science 58, 123-132.

Woudenberg-van, O.M., van, R.F., Sheldon, R.A., 1996. Regioselective acylation of disaccharides in tert-butyl alcohol catalyzed by Candida antarctica lipase. Biotechnology and Bioengineering 49, 328-333.

Zaks, A., Klibanov, A.M., 1988. Enzymatic catalysis in nonaqueous solvents. Journal of Biological Chemistry 263, 3194-3201. 